\title{
WTP for COVID-19 vaccine in Chile
}

The willingness-to-pay (WTP) for COVID-19 vaccination during the pandemic appears to be high in Chile, according to authors of a study published in Applied Health Economics and Health Policy.

Contingent valuation method analysis and a dichotomous choice survey were used to evaluate the WTP for a COVID-19 vaccine in 531 Chilean adults 18-90 years of age who were enrolled in the study in July and August 2020.

Overall, $73 \%$ of the study population had middle- or high-incomes (mean $\$ 2440^{*}$ per month), $98 \%$ claimed that they were well informed on the COVID-19 pandemic, 78\% claimed that they were risk averse, and $52 \%$ had private health insurance.

Variables which had a significant impact on the probability of paying for a COVID-19 vaccine included vaccine price, co-variable income, education level, and the having relatives with COVID-19 infection.

The WTP for a COVID-19 vaccine was up to $\$ 232$.

"Based on the findings in this study, the government could provide the vaccine for free to low-income groups and allow high income groups to purchase the vaccine in the private sector. Additionally, any vaccination campaign design should focus on influencing education through information and increasing the vaccine's acceptance rate, while considering the country's public health situation," said the investigators.

* 2020 US dollars

Cerda AA, et al. Willingness to Pay for a COVID-19 Vaccine. Applied Health Economics and Health Policy : 23 Feb 2021. Available from: URL: https://doi.org/10.1007/ s40258-021-00644-6 